\title{
Long-term outcomes of prenatally diagnosed ventriculomegaly - 10 years of Polish tertiary centre experience
}

\author{
Michal Lipa, Przemyslaw Kosinski, Kamila Wojcieszak, Aleksandra Wesolowska-Tomczyk, \\ Adrianna Szyjka, Martyna Rozek, Miroslaw Wielgos
}

1st Chair and Department of Obstetrics and Gynecology, Medical University of Warsaw, Poland

\begin{abstract}
Objectives: To estimate the prevalence, associated anomalies, and postnatal outcomes in infants prenatally diagnosed with ventriculomegaly.

Material and methods: All cases of ventriculomegaly that were examined and treated by the 1st Department of Obstetrics and Gynecology, at the Medical University of Warsaw, from August 2007 until November 2017 were included in this study. Ultrasound data, and information on perinatal outcomes and long-term postnatal follow up were retrospectively collected by a standardised telephone survey. Ventriculomegaly was diagnosed when the atrial width of the lateral ventricles was $\geq 10 \mathrm{~mm}$. The cases analyzed were divided into two subgroups: isolated ventriculomegaly (IVM) and non-isolated ventriculomegaly (NIVM). Neurodevelopmental complications were differentiated as either moderate or severe and were compared within each group and between groups.

Results: There were 118 cases of prenatally diagnosed ventriculomegaly. Complete follow up records were collected for 54 cases (45.8\%). IVM was diagnosed in 29/54 (53.7\%) cases, while NIVM was diagnosed in the remaining 25 (46.3\%). The mean ventricular width for IVM was $16.93 \mathrm{~mm}$ (range $10.0 \mathrm{~mm}-73.0 \mathrm{~mm}$ ) and $14.08 \mathrm{~mm}$ (range $9.0 \mathrm{~mm}-27.1 \mathrm{~mm}$ ) for NIVM $(p=0.28)$. The mean gestational age at delivery for the IVM cases was $36+4$ weeks and in the NIVM group $33+4$ weeks $(p=0.022)$. Mild VM (10-12 mm) was diagnosed in 22/54 cases (40.7\%), moderate VM (13-15 mm) in 12/54 (22.3\%) and severe $(\geq 15 \mathrm{~mm})$ in $20 / 54(37 \%)$. Among the infants with IVM the rate of severe medical complications was $29.6 \%(8 / 28)$ and for NIVM 667\% (8/12) $(p=0.041)$. Less severe medical conditions affected $6 / 28$ of the infants with IVM (21.4\%) vs $9 / 12$ NIVM cases $(75 \%)(p=0.012)$.

Conclusions: In terms of prenatal diagnosis, treatment of ventriculomegaly remains challenging due to a lack of specific prognostic factors and the significant risk of neurodevelopmental disorders. Nevertheless, isolated ventriculomegaly has significantly better long-term outcomes compared with non-isolated ventriculomegaly. In our material, the rate of severe neurodevelopmental disorders in the non-isolated ventriculomegaly cases was associated with a $52 \%$ rate of adverse perinatal outcomes. On the other hand, less severe medical conditions occurred in $21.4 \%$ of the infants with IVM and in $75 \%$ of the NIVM cases. Furthermore, obstetrical data suggest that the risks of premature delivery and caesarean section are significantly higher in cases of non-isolated ventriculomegaly.
\end{abstract}

Key words: ventriculomegaly; ultrasound; neurodevelopment

Ginekologia Polska 2019; 90, 3: 148-153

\section{INTRODUCTION}

The measurement of the posterior horns of the lateral ventricles has become an integral part of prenatal ultrasound screening. Ventriculomegaly (VM) is diagnosed when the atrial width is $\geq 10 \mathrm{~mm}$ and it remains one of the most common abnormalities detected prenatally. The prevalence range is 0.3 to $2 \%$ of foetuses [1]. To guard against false posi- tive diagnoses, the wide variety of measurement techniques currently used ought to be replaced with a universal measurement protocol. The diagnosis of fetal ventriculomegaly may be described as either idiopathic or a symptom of genetic, infectious or anatomical disorders. Regardless of the primary cause, two groups may be distinguished: isolated (IVM) if there are no other abnormalities present, and non-isolated 
ventriculomegaly (NIVM) where there are other abnormalities present. To assess the severity of VM cases, most studies distinguish between "mild" (10-12 mm), "moderate" (13-15 $\mathrm{mm}$ ) and "severe" ( $\geq 15 \mathrm{~mm}$ ) ventriculomegaly [2]. Despite many studies undertaken by obstetricians and paediatricians that investigate the natural course of VM, the pregnancy and long-term neurodevelopment outcomes remain unknown in most cases. The prognoses of fetuses with VM are controversial because of the large variations in the study scales and follow-up criteria [1]. In this study, we retrospectively analysed and compared pregnancy outcomes, including long-term follow-up outcomes, in infants prenatally diagnosed with IVM and NIVM.

\section{MATERIAL AND METHODS}

The total number of cases $(n=118)$ that were diagnosed with VM and examined in the $1^{\text {st }}$ Department of Obstetrics and Gynecology, Medical University of Warsaw (Poland) from August 2007 until November 2017 were included in this study. We retrospectively collected ultrasonographic data, and information on perinatal outcomes and long-term postnatal follow up. The ultrasound scans were performed by experienced senior ultrasonographers using the Aloka Prosound Alfa 10 system with transabdominal convex probe and the General Electric Voluson E6 BT13.05 system with transabdominal volumetric probe. VM was diagnosed when the atrial width of the lateral ventricle was $\geq 10 \mathrm{~mm}$. Once VM was diagnosed, a detailed scan was performed to exclude associated intracranial or extracranial anomalies. The management protocol also included determination of gestational age, measurement of the contralateral ventricle (in cases of an asymmetrical VM, the larger diameter was considered), placental site, amniotic fluid volume and estimated fetal weight (according to the Hadlock formula). The cases were classified as "Isolated Ventriculomegaly" (IVM) if no associated anomaly was detected, or as "Non-Isolated Ventriculomegaly" (NIVM) which was defined as ultrasound findings of ventriculomegaly with other structural abnormalities or positive findings in TORCH screenings or karyotype examinations.

Medical records of all cases were carefully reviewed. Telephone interviews were used to obtain information about pregnancy outcomes and long-term neurodevelopmental follow-up. All parents were asked questions based on the same questionnaire that included seeking information on the children's: growth, neurological status, developmental status, locomotor activities, coordination, hearing and visual function, speech and socialisation capacities. Information on any current medical treatment or special medical care was also gathered.

Neurodevelopment disorders were divided into moderate and severe and compared within and between the

\begin{tabular}{|c|c|c|}
\hline $\begin{array}{l}\text { System } \\
\text { affected }\end{array}$ & $\begin{array}{l}\text { Moderate } \\
\text { symptoms }\end{array}$ & Severe symptoms \\
\hline \multirow{4}{*}{ 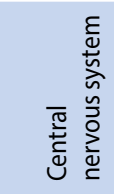 } & \multirow{4}{*}{$\begin{array}{l}\text { Autism spectrum } \\
\text { disorders }\end{array}$} & Tetraplegia \\
\hline & & Epilepsy \\
\hline & & Hydrocephalus \\
\hline & & Microcephaly \\
\hline \multirow{4}{*}{ 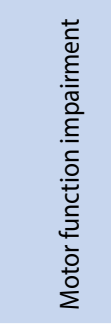 } & $\begin{array}{l}\text { Increased muscle } \\
\text { tension }\end{array}$ & $\begin{array}{l}\text { Delayed psychomotor } \\
\text { development }\end{array}$ \\
\hline & $\begin{array}{l}\text { Reduced muscle } \\
\text { tension }\end{array}$ & \multirow{3}{*}{ Severe paralysis of the limbs } \\
\hline & $\begin{array}{l}\text { Fine motor skills } \\
\text { disorders }\end{array}$ & \\
\hline & $\begin{array}{l}\text { Flaccid paralysis of } \\
\text { the limbs }\end{array}$ & \\
\hline \multirow{3}{*}{ 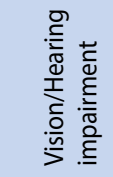 } & \multirow{3}{*}{ Nystagmus } & Retinopathy \\
\hline & & Hearing impairment \\
\hline & & Speech impairment \\
\hline
\end{tabular}

IVM and NIVM groups. The types of moderate and severe disorders analysed in this paper are presented in Table 1.

Follow-up information and obstetrical data such as gestational age at the delivery, method of delivery and birthweight were analysed using SPSS 15.0 (SPSS Inc., Chicago, IL, USA). The chi-square test was applied, and differences were considered to be statistically significant at $p<0.05$. Descriptive statistics were also used for analysis of ultrasonography details and the follow-up characteristics of all the cases.

\section{RESULTS}

Complete records were collected in 54 cases ( $45^{\prime} 8 \%$ ). The high number of incomplete long-term follow-up data was due to inaccurate contact details in the records (i.e., either incorrect or missing telephone numbers). The mean maternal age at the time of the ultrasound scan was 30 years (range 20-45 years). Among the group of 54 fetuses affected with VM, 41/54 cases were singleton pregnancies (75.9\%) and 13/54 in twins (24.1\%). The mean gestational age at the time of referral/diagnosis was $27^{+0}$ weeks (range $18^{+0}-39^{+2}$ weeks). The study group was divided into two subgroups: IVM (isolated ventriculomegaly) and NIVM (non-isolated ventriculomegaly). The IVM group consisted of $29 / 54$ fetuses $(53.7 \%$ ) and the NIVM group consisted of $25 / 54$ cases (46.3\%) (Fig. 1). The average gestational age at the time of diagnosis/counselling for IVM was $27^{+4}$ weeks (range: $18^{+6} 39^{+2}$ ) and for NIVM, $26^{+1}$ weeks (range: $18^{+6}$ $\left.39^{+0}\right)$.

The mean ventricular width for IVM was $16.93 \mathrm{~mm}$ (range $10.0 \mathrm{~mm}-73.0 \mathrm{~mm}$ ) and for NIVM, $14.08 \mathrm{~mm}$ (range $9.0 \mathrm{~mm}$ - 


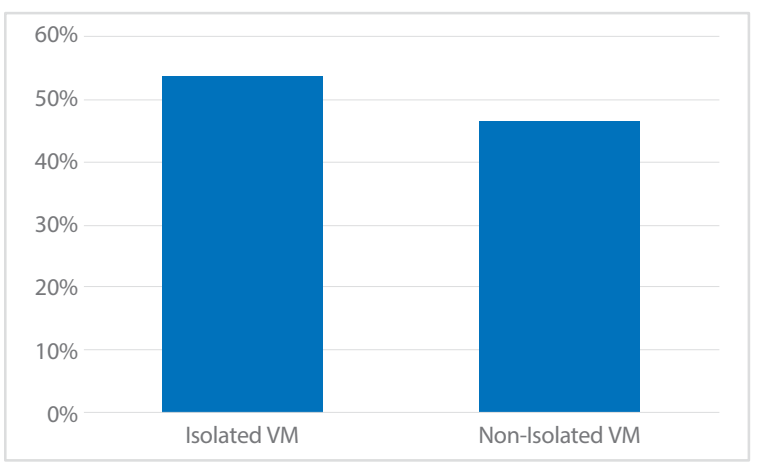

Figure 1. Prevalence of isolated vs. non-isolated ventriculomegaly in the study group

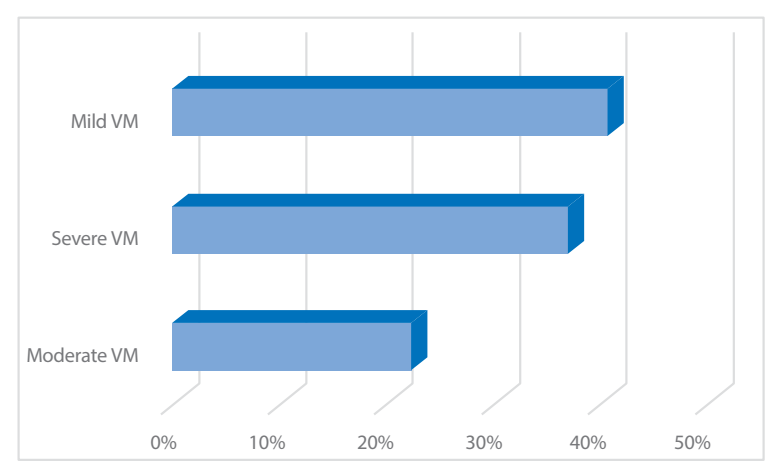

Figure 2. Ventriculomegaly severity range in the study group. (Mild VM: 10-12 mm; moderate VM: 13-15 mm; severe VM: $\geq 15 \mathrm{~mm}$ )

$27.1 \mathrm{~mm})(p=0.28)$. Overall, mild VM $(10-12 \mathrm{~mm})$ was diagnosed in $22 / 54$ cases $(40.7 \%)$, moderate VM (13-15 $\mathrm{mm})$ in $12 / 54$ (22.3\%), and severe ( $\geq 15 \mathrm{~mm}$ ) in 20/54 (37\%) (Fig. 2).

Multiple pregnancy rates were similar in both groups ( $27.6 \%$ vs $20.0 \%$ in IVM and NIVM respectively). The mean gestational age at delivery for IVM was $36^{+4}$ weeks and in the NIVM group $33^{+4}$ weeks $(p=0.022)$. The rate of preterm delivery < 34 weeks in IVM was $6 / 29$ (21\%) and in NIVM 8/25 (32\%), and the difference between these was statistically significant $(p=0.027)$. The mean birth weight for IVM neonates was $2668 \mathrm{~g}$ vs $2129 \mathrm{~g}$ for the NIVM neonate, which showed a borderline statistical significance $(p=0.08)$.

The most frequent coexisting fetal abnormalities in the NIVM group were agenesis of corpus callosum, spina bifida, hydrocephalus, subarachnoid cyst, omphalocele, ventricular septal defect, diaphragmatic hernia, duodenal atresia, mega cisterna magna, talipes, kidney agenesis, and small for gestational age. There was one case of trisomy 13 (classified as NIVM).

Caesarean section rates in both groups were high amounting to $79.3 \%$ in the IVM group vs $77.3 \%$ in the NIVM group. The NIVM group was associated with a $52 \%$ rate of adverse perinatal outcomes. In 25 of the NIVM cases there were reported: $8 / 25$ (32\%) postnatal deaths, 2/25 (8\%) stillbirths, and 3/25 (12\%) spontaneous abortions. There was

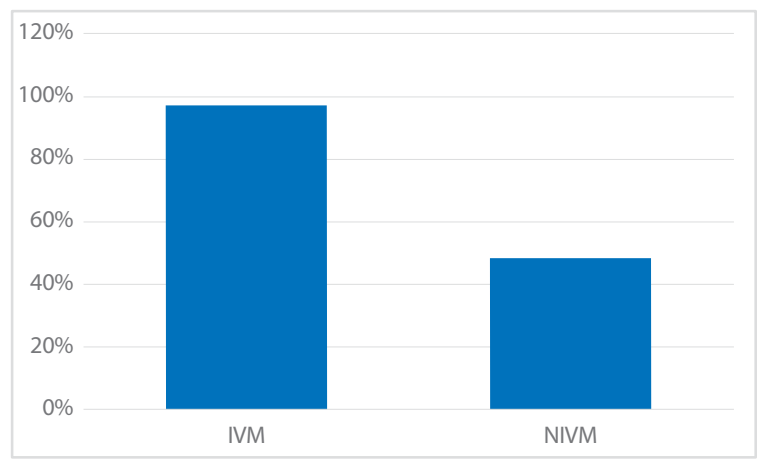

Figure 3. Survivors in the IVM and NIVM groups

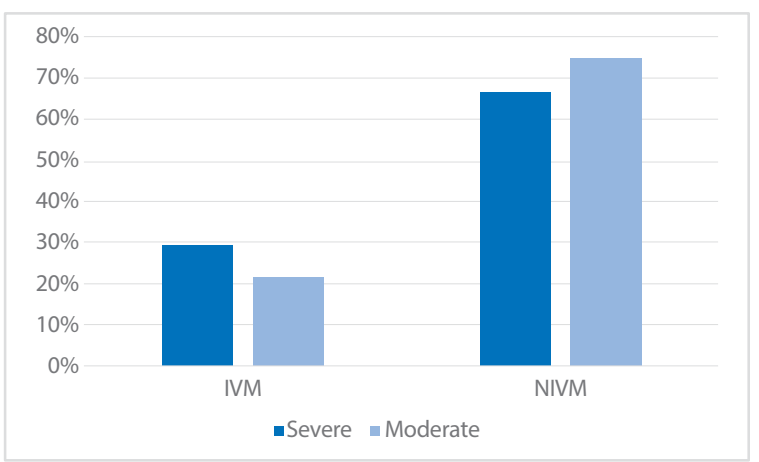

Figure 4. Prevalence of severe and moderate medical conditions diagnosed in the two subgroups

only $1 / 29(3.4 \%)$ postnatal death in the IVM group. A total of 12 of 25 (48\%) NIVM fetuses survived. (Fig. 3)

The average age of the children at the parental telephone interview was 23 months (range 6 weeks to 5 years). Among the surviving IVM infants, the rate of severe neurodevelopmental disorders (epilepsy, tetraplegia, speech or hearing impairment, microcephaly, hydrocephalus, delayed psychomotor development) was $29.6 \%(8 / 28)$, and among the NIVM survivors, $66.7 \%(8 / 12)(p=0.041)$. The medical complications reported in both groups are listed in Table 1. Less severe medical conditions, such as motor function disorders, autism spectrum disorders, or reduced or increased muscle tension affected $6 / 28$ of infants in the IVM group (21.4\%) vs $9 / 12$ of the NIVM cases (75\%), which was statistically significant $(p=0.012)$ (Fig. 4).

\section{DISCUSSION}

The overall frequency of VM in the general population is approximately $0.3-2 \%$. Most authors classify VM according to the three-degree classification, as either "mild" (10-12 mm), "moderate" (13-15 mm) or "severe" ( $\geq 15 \mathrm{~mm}$ ) ventriculomegaly $[2,3]$. In our study group, rates of mild VM $(10-12 \mathrm{~mm})$ and severe VM ( $\geq 15 \mathrm{~mm})$ were almost equal ( $40.7 \%$ and $37 \%$ respectively), while $22.3 \%$ were classified 
as moderate VM (13-15 mm). In a larger group of $241 \mathrm{fe}-$ tuses described by Chu et al. [1], the rate of moderate VM was comparable (23.2\%), but in the same study the rate of mild VM was significantly higher that our result (62.7\%). On the other hand, in that same paper, the rate of NIVM was high and reached $66 \%$ compared with $46.3 \%$ in our study group. Regardless of the severity of VM, follow-up scans are recommended as the risk of progression of ventricular dilatation is about $16 \%$ [4]. In most studies, progression of ventriculomegaly is defined as an increase in the ventricular measurement of more than $3 \mathrm{~mm}$ [5]. Several studies suggested the atrial width may determine the final outcome [6]. However, a large meta-analysis of pooled data did not demonstrate differences in the neurological outcomes between mild and moderate groups [4]. There is also a male predominance among fetuses with a mild VM diagnosis. It is noteworthy, that the female gender correlates significantly with worse neurodevelopmental outcomes [7].

There is wide variation in the reported incidence of neurodevelopmental delay, but several studies suggest this is around $11 \%[4,7]$. Weichert et al. [8] reported poor prognoses in $41 \%$ of cases. Another study reported handicap-free survival in just over one third of cases affected by severe isolated VM [9]. According to several other papers, a ventricular atrial width of $>15 \mathrm{~mm}$ is related to poor outcomes $[6,10,11]$. A large systematic review and meta-analysis revealed abnormal neurodevelopmental outcomes in $7 \%$ and $8 \%$ of mild and moderate VM cases, respectively, and in $58 \%$ of severe VM cases, suggesting the rate of neurodevelopmental delay for isolated mild and moderate VM is comparable to that of the general population [12].

We analysed 54 cases of VM diagnosed prenatally: 41 singleton (75.9\%) and 13 twin pregnancies (24.1\%). In a larger study group of 241 fetuses affected with VM, the multiple pregnancy rate was significantly lower than in our study — only $1.6 \%$ were twins [1]. The high incidence of multiple pregnancies in our group may be associated with the specific profile of the patients, because most of them were referred to a tertiary ultrasound department for an expert opinion. Moreover, many of the twin pregnancies in our study group remained under prenatal surveillance in the Multiple Pregnancies Out Patient Unit in our Department.

The mean maternal age in our study was 30 years, which was similar to that in the Gomez et al. study (29 years) [13]. The average gestational age at the time of delivery in the IVM group was significantly higher compared with that of the NIVM group $\left(36^{+4}\right.$ weeks vs. $33^{+4}$ weeks, respectively). This was most likely due to accompanying fetal abnormalities that increase the risk of preterm delivery or premature rupture of membranes (PROM), such as polyhydramnios in the course of abnormal fetal swallowing associated with central nervous system defects. Discordant rates of preterm deliveries resulted in the differences between the mean birth weights ( $2668 \mathrm{~g}$ vs $2129 \mathrm{~g}$ for the IVM and NIVM groups, respectively). Furthermore, there were surprisingly high rates of caesarean section in both groups - 79.3\% in IVM and 77.3\% in NIVM. A prevalence of caesarean deliveries in VM cases has also been reported by other authors. Hannon et al. [10] found an association between the final atrium measurement and mode of delivery with the odds of elective cesarean delivery being increased by $11 \%$ for each additional millimetre in atrial width. The most frequent indications for caesarean section in our group were: failure to progress, malpresentation, cephalopelvic disproportion, placenta praevia and hypertensive disorders. It must be underscored that the VM itself is not an indication for caesarean section. Pisapia et. al. [14] revealed that head circumference $(\mathrm{HC})$ remains normal in most cases of VM. Only in cases with severe hydrocephaly causing abnormal intracranial pressure, resulting in increased head circumference $(\mathrm{HC})$ above $400 \mathrm{~mm}$, may qualify for elective caesarean section due to the high risk of obstetrical complications; however, there were no such cases in our study group.

The main goal of our study was to investigate the long-term neurological development outcomes following prenatal diagnosis of ventriculomegaly. In the group of infants with IVM, the rate of severe medical complications (epilepsy, tetraplegia, speech or hearing impairment, microcephaly, hydrocephalus or delayed psychomotor development) was $29.6 \%$ (8/28), while in the NIVM group the rate was $66.7 \%$ $(8 / 12)$. We found a stronger association of poor prognosis with coexisting fetal abnormalities (NIVM) rather than with atrial width alone (the mean width for IVM was $16.93 \mathrm{~mm}$ and $14.08 \mathrm{~mm}$ for NIVM; $\mathrm{p}=0.28$ ). In the literature, mortality and impaired neurodevelopment in infants affected with VM differs between obstetrical and paediatric studies. Moreover, neurodevelopmental delay in preschool children is not rare, however reliable data on its precise prevalence are limited. In almost $10 \%$ of cases, associated anomalies are diagnosed in the postnatal period. Most infants with a prenatal diagnosis of IVM have normal neurological development at least in infancy and only $11 \%$ present some abnormalities [4].

Despite numerous studies, the perinatal outcomes and early adolescence neurodevelopment of infants affected with prenatally diagnosed VM remain unknown. Several studies investigating perinatal outcomes in cases affected with VM diagnosed prenatally revealed that any associated defect was related to significantly worse outcomes. The livebirth rates in NIVM cases is about $30-40 \%[10,11]$. This is consistent with our data, as a total of 12 out of 25 (48\%) NIVM fetuses survived. On the other hand, in our IVM group, neonatal death occurred in only $3.4 \%$ of cases. The high rate of adverse perinatal outcomes in our material may be associated with the low number of pregnancy terminations (TOP) and the decision of patients to continue the gestation. 
A major difficulty in prenatal diagnosis and treatment is related to the lack of a standardized and universal definition. A ventricular diameter of $10 \mathrm{~mm}$ correlates with more than two standard deviations of the normal, hence it qualifies as ventriculomegaly with most authors $[15,16]$. Unfortunately, some clinicians tend to use the terms "ventriculomegaly" and "hydrocephalus" synonymously. However, even severe VM and the hydrocephalic state should be clearly differentiated. Besides significant dilatation of the ventricular system, hydrocephaly is associated with increased intracranial pressure. Unfortunately, though it is not possible to measure it antenatally, some indirect signs, such as dangling choroid plexuses or decreased volume and abnormal vascular flow pattern in subarachnoid vessels, may help achieve a differential diagnosis. Therefore, if there is no sign of increased intracranial pressure, the term "ventriculomegaly" should be used rather than "hydrocephalus". In selected cases, fetal MRI may have an additional diagnostic value; however, according to Malinger et. al., [17] a neurosonogram scan performed by an experienced sonographer has a similar accuracy to an MRI. In most cases neurosonography seems sufficient, especially with a transvaginal approach and vertex presentation, but MRI might be an extremely helpful imaging method in cases of poor visualisation or when a more detailed differential diagnostic tool is needed [15]. On the other hand, a large study involving third trimester MRI scans of 185 fetuses with moderate VM revealed information relevant enough to modify the obstetric management in $6 \%$ of cases [18]. In all cases of ventriculomegaly, the best available advanced neuroimaging should be offered as neurosonography has is highly accurate in the prenatal diagnosis of central nervous system abnormalities. It is noteworthy that approximately $40-60 \%$ of cases of ventriculomegaly have associated CNS or extra-CNS abnormalities $[1,7,19,20]$. This is consistent with our data - NIVM were diagnosed with CNS or extra-CNS abnormalities in $25 / 54$ cases (46.3\%). There are several coexisting conditions leading to VM: meta-analysis by Gaglioti et al. [11] from 2009 points that abnormal turnover of cerebrospinal fluid (CSF), or congenital central nervous system defects such as agenesis of the corpus callosum, neuronal migration disorders, schizencephaly, vertical transmission of cytomegalovirus (CMV) or toxoplasmosis, intracranial haemorrhage and defects of the cerebral vessels may result in dilatation of the ventricular system. According to the literature, $9-12 \%$ of ventriculomegaly cases have an abnormal karyotype [1, 21]. On the other hand, a study by Melchiorre et al. [4] reports the rate of chromosomal abnormalities as $2.8 \%$. In our group, there was only one case of trisomy 13 . In the Melchiorre et al study, the group was screened for chromosomal abnormalities in the first trimester; whereas in our study, the single case of trisomy 13 was detected in the second trimester of pregnancy due to coexisting fetal abnormalities.

\section{Study limitations}

Several limitations of our study should be considered. The study was a retrospective analysis based on ultrasound reports, obstetrical data and paediatric follow-up. A precise protocol for interviewing parents by telephone was applied in every case. Most likely, in the instance of a higher rate of postnatal follow-up our results could have shed more light on the prenatal diagnosis of VM. Moreover, the paediatric follow-up was not evaluated by a paediatric neurologist and the infants' ages at the time of the telephone interview ranged from 6 weeks to 5 years. Considering the psychological impact of offspring's disease some of the data may be biased or inconclusive. On the other hand, this is the normal evaluation route from prenatal diagnosis to postnatal follow-up with retrospective studies.

\section{CONCLUSIONS}

Heterogenous aetiology and associated disorders suggest that in many cases VM should be considered as a symptom, not a disease itself. Despite the growing availability of different imaging techniques, a detailed ultrasound scan remains the first step and a reliable diagnostic tool. It has to be underscored that the measurement technique used plays a crucial role in the diagnostic process. Measurement of the atrium of the lateral ventricles on an axial transventricular plane, including the frontal horns and the cavum septi pellucidi, with symmetrical hemispheres is considered to be the most accurate [22]. Guidelines published by the International Society of Ultrasound in Obstetrics and Gynecology are based on the protocol by L. Guibaud, and this seems to provide the highest intra-observer reproducibility [23]. Once VM is diagnosed prenatally, associated CNS defects should be excluded. The most common associated CNS defects are agenesis of the corpus callosum (ACC) and spina bifida.

Due to unknown causes, prediction of postnatal neurodevelopment in fetuses affected with VM diagnosed prenatally is extremely unpredictable. Unfortunately, the results of studies in both obstetrical and paediatric fields are inconclusive. We are not able to precisely predict long-term follow up in individual cases. The available severity classifications and differentiating IVM from NIVM may help in prenatal counselling. Most of our results were like those available in the literature. Nonetheless, the obstetrical and paediatric data collected suggests that only a comprehensive prenatal diagnostic approach will help to determine the pregnancy outcome in each case. 


\section{REFERENCES}

1. Chu N, Zhang Y, Yan Y, et al. Fetal ventriculomegaly: Pregnancy outcomes and follow-ups in ten years. Biosci Trends. 2016; 10(2): 125-132, doi: 10.5582/bst.2016.01046, indexed in Pubmed: 27087461.

2. Kramer RL, Yaron Y, Johnson MP, et al. Differences in measurements of the atria of the lateral ventricle: does gender matter? Fetal Diagn Ther. 1997; 12(5): 304-305, doi: 10.1159/000264492, indexed in Pubmed: 9430215.

3. Signorelli $M$, Tiberti $A$, Valseriati $D$, et al. Width of the fetal lateral ventricular atrium between 10 and $12 \mathrm{~mm}$ : a simple variation of the norm? Ultrasound Obstet Gynecol. 2004; 23(1): 14-18, doi: 10.1002/uog.941, indexed in Pubmed: 14970992.

4. Melchiorre $\mathrm{K}$, Bhide $\mathrm{A}$, Gika AD, et al. Counseling in isolated mild fetal ventriculomegaly. Ultrasound Obstet Gynecol. 2009; 34(2): 212-224, doi: 10.1002/uog.7307, indexed in Pubmed: 19644944.

5. Ouahba J, Luton $D$, Vuillard $E$, et al. Prenatal isolated mild ventriculomegaly: outcome in 167 cases. BJOG. 2006; 113(9): 1072-1079, doi: 10. 1111/j.1471-0528.2006.01050.x, indexed in Pubmed: 16956339.

6. Breeze ACG, Dey PK, Lees CC, et al. Obstetric and neonatal outcomes in apparently isolated mild fetal ventriculomegaly. J Perinat Med. 2005; 33(3): 236-240, doi: 10.1515/JPM.2005.043, indexed in Pubmed: 15914347.

7. Pilu G, Falco P, Gabrielli S, et al. The clinical significance of fetal isolated cerebral borderline ventriculomegaly: report of 31 cases and review of the literature. Ultrasound Obstet Gynecol. 1999; 14(5): 320-326, doi: 1 0.1046/j.1469-0705.1999.14050320.x, indexed in Pubmed: 10623991.

8. Weichert J, Hartge D, Krapp M, et al. Prevalence, characteristics and perinatal outcome of fetal ventriculomegaly in 29,000 pregnancies followed at a single institution. Fetal Diagn Ther. 2010; 27(3): 142-148, doi: 10.1159/000304735, indexed in Pubmed: 20339298.

9. Carta S, Kaelin Agten A, Belcaro C, et al. Outcome of fetuses with prenatal diagnosis of isolated severe bilateral ventriculomegaly: systematic review and meta-analysis. Ultrasound Obstet Gynecol. 2018; 52(2): 165-173, doi: 10.1002/uog.19038, indexed in Pubmed: 29484752.

10. Hannon T, Tennant PWG, Rankin J, et al. Epidemiology, natural history, progression, and postnatal outcome of severe fetal ventriculomegaly. Obstet Gynecol. 2012; 120(6): 1345-1353, doi: http://10.1097/AOG.0b013e3182732b53, indexed in Pubmed: 23168759.

11. Gaglioti P, Danelon D, Bontempo S, et al. Fetal cerebral ventriculomegaly: outcome in 176 cases. Ultrasound Obstet Gynecol. 2005; 25(4): 372-377, doi: 10.1002/uog.1857, indexed in Pubmed: 15791694.

12. Pagani G, Thilaganathan B, Prefumo F. Neurodevelopmental outcome in isolated mild fetal ventriculomegaly: systematic review and meta-analysis. Ultrasound Obstet Gynecol. 2014; 44(3): 254-260, doi: 10.1002/uog.13364, indexed in Pubmed: 24623452.

13. Gómez-Arriaga P, Herraiz I, Puente JM, et al. Mid-term neurodevelopmental outcome in isolated mild ventriculomegaly diagnosed in fetal life. Fetal Diagn Ther. 2012; 31(1): 12-18, doi: 10.1159/000331408, indexed in Pubmed: 22178749.

14. Pisapia JM, Sinha S, Zarnow DM, et al. Fetal ventriculomegaly: Diagnosis, treatment, and future directions. Childs Nerv Syst. 2017; 33(7): 11131123, doi: 10.1007/s00381-017-3441-y, indexed in Pubmed: 28510072.

15. Prayer D, Paladini D, Deprest J. Should MRI be performed on all fetuses with mild ventriculomegaly? Prenat Diagn. 2019 [Epub ahead of print], doi: 10.1002/pd.5416, indexed in Pubmed: 30614017.

16. International Society of Ultrasound in Obstetrics \& Gynecology Education Committee. Sonographic examination of the fetal central nervous system: guidelines for performing the,basic examination' and the, fetal neurosonogram'. Ultrasound Obstet Gynecol. 2007; 29(1): 109-116, doi: 10.1002/uog.3909, indexed in Pubmed: 17200992.

17. Malinger G, Paladini D, Pilu G, et al. Fetal cerebral magnetic resonance imaging, neurosonography and the brave new world of fetal medicine. Ultrasound Obstet Gynecol. 2017; 50(6):679-680, doi: 10.1002/uog.17538, indexed in Pubmed: 28568972.

18. Salomon LJ, Ouahba J, Delezoide AL, et al. Third-trimester fetal MRI in isolated 10- to 12-mm ventriculomegaly: is it worth it? BJOG. 2006; 113(8): 942-947, doi: 10.1111/j.1471-0528.2006.01003.x, indexed in Pubmed: 16827833

19. Senat MV, Bernard JP, Schwärzler P, et al. Prenatal diagnosis and follow-up of 14 cases of unilateral ventriculomegaly. Ultrasound Obstet Gynecol. 1999; 14(5): 327-332, doi: 10.1046/j.1469-0705.1999.14050327.x, indexed in Pubmed: 10623992.

20. Vergani P, Locatelli A, Strobelt N, et al. Clinical outcome of mild fetal ventriculomegaly. Am J Obstet Gynecol. 1998; 178(2): 218-222, indexed in Pubmed: 9500477.

21. Bromley B, Frigoletto F, Benacerraf B. Mild fetal lateral cerebral ventriculomegaly: Clinical course and outcome. American Journal of Obstetrics and Gynecology. 1991; 164(3): 863-867, doi: 10.1016/0002 9378(91)90530-5.

22. Pilu G, Hobbins JC. Sonography of fetal cerebrospinal anomalies. Prenat Diagn. 2002; 22(4): 321-330, doi: 10.1002/pd.310, indexed in Pubmed: 11981913.

23. Guibaud L. Fetal cerebral ventricular measurement and ventriculomegaly: time for procedure standardization. Ultrasound Obstet Gynecol. 2009; 34(2): 127-130, doi: 10.1002/uog.6456, indexed in Pubmed: 19644945. 